\title{
Parental mosaicism of JAG1 mutations in families with Alagille syndrome
}

Joannis Giannakudis ${ }^{*, 1}$, Albrecht Röpke ${ }^{1}$, Annegret Kujat ${ }^{1}$, Malgorzata Krajewska-Walasek ${ }^{2}$, Helen Hughes ${ }^{3}$, Jean-Pierre Fryns ${ }^{4}$, Agnes Bankier ${ }^{5}$, David Amor ${ }^{5}$, Mike Schlicker ${ }^{1}$ and Ingo Hansmann ${ }^{1}$

${ }^{1}$ Institut für Humangenetik und Medizinische Biologie, University Halle-Wittenberg, Halle, Germany; ${ }^{2}$ Department of Medical Genetics, The Children's Memorial Health Institute, Warsaw, Poland; ${ }^{3}$ Institute of Medical Genetics, University Hospital, Cardiff, UK; ${ }^{4}$ Center for Human Genetics, University of Leuven, Leuven, Belgium; ${ }^{5}$ Victorian Clinical Genetics Service, Royal Children's Hospital, Melbourne, Australia

The Alagille syndrome (AGS), a congenital disorder affecting liver, heart, skeleton and eye in association with a typical face, is an autosomal dominant disease with nearly complete penetrance and variable expression. AGS is caused by mutations in the developmentally important JAG1 gene. In our mutation screening, where 61 mutations in JAG1 were detected, we identified five cases where mosaicism is present. Our results point to a significant frequency of mosaicism for JAG1 mutations in AGS of more than $\mathbf{8 . 2 \%}$. Because mosaicism may be associated with a very mild phenotype, the appropriate diagnosis of AGS and consequently the determination of the recurrence risk can be complicated. European Journal of Human Genetics (2001) 9, 209-216.

Keywords: Alagille syndrome; JAG1 mutation; mosaicism; mild phenotype; chromosome 20

\section{Introduction}

The Alagille syndrome (AGS, MIM 118450) is an autosomal dominant disorder which displays five major symptoms: cholestasis, peripheral pulmonic stenosis, butterfly vertebrae, eye abnormality, (eg posterior embryotoxon) and a characteristic face. Several other features including kidney cysts and hearing loss have been reported as well. AGS occurs with a minimal estimated frequency of 1:70000 and is one of the main causes for chronic liver disease in infancy. AGS is predominantly the result of mutations in JAG1 (55-70\%) while only $30-45 \%$ of the cases are inherited. ${ }^{1}$ Because AGS presents with a highly variable expressivity and nearly complete penetrance ${ }^{2}$ the diagnosis can be difficult. According to Alagille et $\mathrm{al}^{3}$ the diagnostic criteria may comprise

*Correspondence: J Giannakudis, Institut für Humangenetik und Medizinische Biologie, University Halle-Wittenberg, Magdeburgerstr. 2, D-06097 Halle, Germany. Tel: +49 345557 4413; Fax: +49 557 4293; E-mail: joannis.giannakudis@medizin.uni-halle.de

Received 8 August 2000; revised 15 November 2000; accepted 20 November 2000 three of the five major symptoms or only two symptoms provided that the patient has a positive family history. ${ }^{4}$ The AGS locus was initially mapped by Byrne et al to 20p11.2 and refined by Schnittger et $a l^{6}$ to 20p11.23-p12.1 by analysing a patient with an interstitial deletion. Subsequently, by analysis of further deletion cases and linkage studies this assignment was confirmed. ${ }^{7-9}$ Recently, the gene Jagged1 (JAG1) was identified that maps to the AGS critical region. ${ }^{10-12}$ JAG1 encodes a transmembrane ligand for the Notch receptors which take part in cell signalling and play a fundamental role in developmentally important processes like cell fate determination in many organisms. ${ }^{13}$ Several mutations including small deletions, insertions, splice site, missense variations ${ }^{11,12,14-16}$ and a paracentric inversion disrupting the gene (Stankiewicz 1997, personal communication) have been reported defining JAG1 as the AGS gene.

During the course of our mutation analysis in AGS patients one family presented where two children were shown to carry a 4-base-pair insertion in the JAG1 gene. Sequence analysis of either parent's DNA failed to detect this mutation. However, by designing a mutation specific PCR primer which at the $3^{\prime}$ 
end corresponds to the inserted sequence, the mother was found to carry the same mutation. This observation raised the question for mosaicism in AGS. In this paper we have examined type and frequency of mosaicism and provide evidence that this genetic peculiarity can be associated with a mild phenotype. Thus, mosaicism in AGS has to be taken into account in the clinical diagnosis as well as in the determination of the recurrence risk in genetic counselling.

\section{Material and methods AGS patients}

Most of our patients were referred from their physicians and were included in our analysis if they met the diagnostic criteria for AGS as described by Alagille et $a l^{3}$ and Elmslie et al. ${ }^{4}$ In addition the physicians were requested to fill in a questionnaire in order to standardise the clinical evaluation of characteristic symptoms. In this questionnaire all five major symptoms and some minor features and their severity were asked for and were stated as present $(+)$ or absent $(-)$. In case no information could be provided the respective feature was ticked as not reported (NR).

\section{Fluorescence in situ hybridisation}

Fluorescence in situ hybridisation (FISH) was essentially performed according to a modified procedure of Pinkel et $a l^{17}$ using cosmid 3e9 that carries the entire JAG1 gene.
Monochromatic images were captured using an Axiophot microscope (Carl Zeiss, Oberkochen, Germany) equipped with a CCD-camera and were pseudocoloured using ISIS software from MetaSystem.

\section{PCR and primer sequences}

PCR conditions for SSCP and microsatellite routine analysis and primer sequences are described elsewhere. ${ }^{12}$ In order to exclude preferential misamplification of one allele due to polymorphic sequences encompassing the primer region, up to three different primer pairs for a specific locus were chosen (Figure 1). PCR reactions were repeated more than three times after subjecting the target DNA to purification procedures including desalting and/or phenol extraction.

\section{Single-strand conformational polymorphism (SSCP)}

SSCP was carried out on an ALFexpress system (Pharmacia Biotech) using two Cy5-fluorescence labelled exon specific primers. Electrophoresis was performed at 18 and $10^{\circ} \mathrm{C}$, respectively. Band shifts were subjected to sequencing according to the Thermo Sequenase II dye terminator cycle sequencing technique (Amersham Pharmacia Biotech).

\section{Microsatellite analysis}

Deletion screening was done using microsatellite marker D20S1154 from intron 19 of the JAG1 gene. ${ }^{12}$ Genotyping case 1)

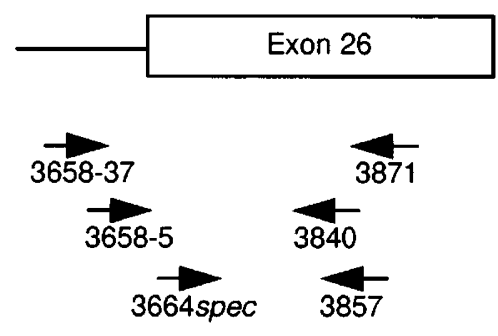

case 3)

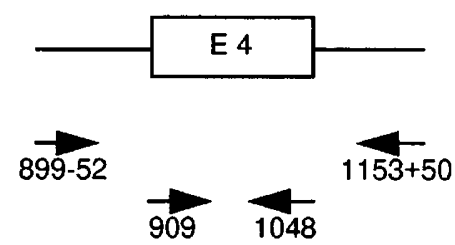

case 2)

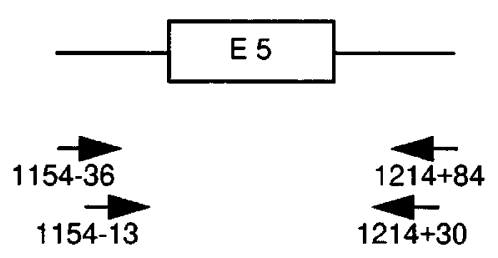

case 4)

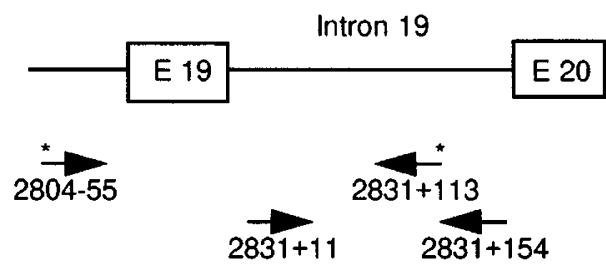

Figure 1 Relative position of primer pairs used to amplify mosaic mutations in AGS patients. Numbers below arrows depict nucleotide position relative to JAG1 cDNA. Figure not drawn to scale. Sequences according to Oda et al ${ }^{12}$ except ${ }^{*}$ : according to Li et al; ${ }^{11}$ case 4 intron 19 primer represent the polymorphic marker D20S1154 used for deletion detection; ins: primer carrying mutation specific insertion of 4 nucleotides; E: exon. 
was performed on an ALFexpress using one Cy5-fluorescence labelled primer.

Paternity testing of the pedigree case 5 was performed using microsatellite markers D2S2393, D5S630, D17S1566, D18S869 and D20S1154.

\section{Detection of mosaicism}

Mosaicism was assessed mainly if a significant signal reduction in SSCP-, restriction-, microsatellite- and/or sequence analysis was observed in comparison to a control reaction.

\section{Results}

During the course of our mutation analysis in the JAG1 coding region we observed mosaicism for a JAG1 mutation in the mother of two affected sisters with the same mutation. Subsequently, we reviewed 61 AGS cases where mutations had been identified by us (in preparation) particularly focusing on mosaicism. Parental mosaicism was assessed in all AGS cases where parental DNA was at our disposal. Briefly, in 51 of 61 cases where parental DNA was at our disposal, we previously identified 32 mutations (63\%) to have occurred de novo and 19 mutations (37\%) that have been inherited from the parents. After reviewing we were able to determine three further cases of parental mosaicism together reflecting $7.8 \%$ (4/51) which changed the ratio of de novo vs inherited mutations to $57: 43 \%$. One case was observed where mosaicism was present in the patient himself. Altogether mosaicism was detected in five cases reflecting an overall frequency of $8.2 \%$.

\section{Case 1}

A family is presented with three children, two of them with a clinically well defined AGS. The mother exhibits only a characteristic face in combination with an embryotoxon posterior but no other features of AGS. SSCP analysis of both daughters showed an unusual pattern of the exon 26 PCR product. Sequence analysis lead to the identification of a four-nucleotide insertion (3681insTGAG) in both daughters but no abnormality was detected in either parent's DNA (not shown). To re-analyse the parents, a new forward primer within the exon 26 amplicon was designed which at the $3^{\prime}$ end carries the four inserted nucleotides (Table 1). A PCR using the insertion specific forward primer resulted in a mutation specific DNA product amplified from the DNA of the two daughters as well as from the mother, yet in the latter with a low fluorescence intensity (Figure 2).

A)
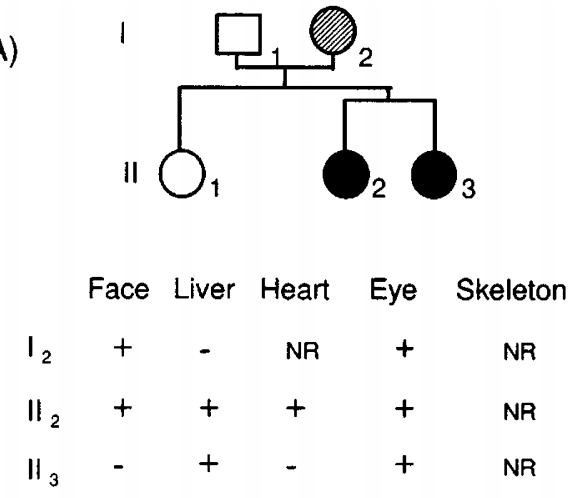

B)

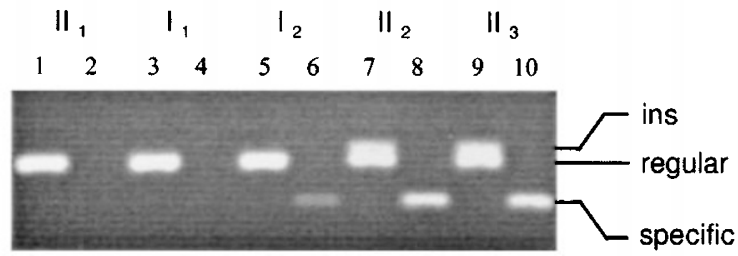

Figure 2 Case 1 (3681 ins4). (A) AGS family and symptoms of the affected individuals. Hatched symbol: mosaicism for the 3681 ins4 mutation; NR: not reported. (B) Lanes 1, 3, 5, 7, 9: amplification product of exon 26 using normal primers and 2, 4, $6,8,10$ using mutation specific primers. Lanes 1,2: unaffected daughter; 3, 4 unaffected father; 5, 6: mother; 7, 8 and 9, 10 affected daughters, respectively; ins: mutation relevant fragments appear with normal primers only in lanes 7 and 9; regular: fragment corresponding to normal allele; specific: using mutation specific primers the relevant fragments appear in the affected children's and faintly in the mother's DNA, lanes 8, 10 and 6 , respectively.

Table 1 Primer sequences used to demonstrate mosaicism in JAG1

\begin{tabular}{|c|c|c|c|c|}
\hline Case & $\begin{array}{l}5^{\prime} \text {-position } \\
\text { relative to cDNA }\end{array}$ & Forward primer & $\begin{array}{l}3 \text {-position } \\
\text { relative to } C D N A\end{array}$ & Reverse primer \\
\hline \multirow[t]{3}{*}{1} & $3658-37$ & TCTTGGAGAGTTAATTGGTTTTGTGC* & 3871 & CCTTGATGGGGACCGTGTTG* \\
\hline & $3658-5$ & TACAGATTTCCTTGTTCCCTTG & 3840 & TCAATGGGGTTTTTGATCTG \\
\hline & 3664 & TTGTTCCCTTGCTGAGTGAG & 3857 & GTGTTGGCCCCATGTTTC \\
\hline \multirow[t]{2}{*}{2} & $1154-36$ & GCAGTGTGCTGACACGCCCT* & $1214+84$ & AAGAGGCATAGTCACAATAAAGTCAGTTCC* \\
\hline & $1154-13$ & СTGTTTTTTACAGCTATTTGCCG & $1214+30$ & AGAAATCTCACAAAAGACCAGTTG \\
\hline \multirow[t]{2}{*}{3} & $899-52$ & GGGAAGAAGGCTGCAATGTGAATA* & $1153+50$ & GACACTAAAAGCAACAGGCACACG* \\
\hline & 909 & TATTGAAAAGGCTTCTCACTCG & 1048 & TGCAGCCAAAGCCATAGTAG \\
\hline \multirow[t]{2}{*}{4} & $2804-55$ & АСTTGCATTTTAАAСАСААТСССТG & $2831+113$ & CTAAGACCGCTTTCCCTGTTGA \\
\hline & $2831+11$ & ACATCCTTTTAAGCCAGCAC\# & $2831+154$ & AAAGCAGTCTGAGGCCCC\# \\
\hline
\end{tabular}

*Sequence according to Oda et $a l^{12}$ and \# to Li et al. ${ }^{11}$ Bold: nucleotides corresponding to the four nucleotide insertion of case 1. 


\section{Case 2}

A family with two affected sons and one unaffected daughter. Both sons, one was deceased, exhibited a complete syndrome, ie all five major symptoms were present. The mother presented only the characteristic face and an embryotoxon posterior but no other features. Upon SSCP of the exon 5 PCR product a fragment shift was observed in the son's and very weakly in the mother's lane (Figure 3). Sequence analysis of the exon - intron boundary of the son's DNA showed a splicesite mutation $1214+1 \mathrm{G} \rightarrow \mathrm{A}$ with nearly identical peak heights corresponding to the two nucleotides. In contrast sequencing of the mother's DNA resulted in a reduced signal intensity of the mutated nucleotide indicating unequal amounts of normal vs mutated target alleles. Misamplification of one allele due to polymorphism(s) is unlikely because the PCR result was confirmed using a second and different primer pair (see Material and methods). Therefore we consider this case to represent maternal mosaicism.

\section{Case 3}

An AGS family with one affected son presenting a complete syndrome. The father showed only the characteristic face. Otherwise, he is reported to be clinically normal without any sign of heart, liver, vertebrae or eye abnormality. SSCP analysis showed a fragment shift of the exon 4 in the lane of the affected son but not in either parent's lane. Sequencing of the respective fragment revealed the missense mutation $\mathrm{R} 184 \mathrm{H}$ characterised by a change of nucleotide $\mathrm{G}$ to $\mathrm{A}$ in the son's DNA. Figure $4 \mathrm{C}$ shows the sequence of the antisense strand. Therefore the mutation depicts a change $C$ to $T$. In contrast, the parent's DNA at this cDNA position appeared as normal except for a faint A-nucleotide signal in the sequence of the father. Because this mutation creates a new restriction site for PmlI we subjected the PCR products of exon 4 to digestion in order to exclude a non specific background artefact. We observed a prominent mutation specific fragment in the child's lane and a very weak one in the father's lane (Figure 4). We consider this case to represent paternal mosaicism which in lymphocytes affects $10-20 \%$ of the cells, as estimated from the signal intensity of the mutation specific restriction fragment.

\section{Case 4}

In this family the son presented with a complete AGS syndrome with all five major features. The father as well as the sister were noted as healthy, whereas the mother
A)

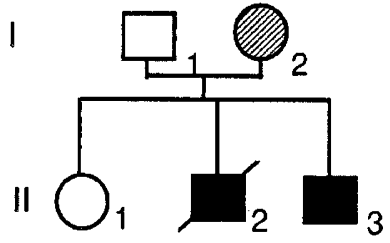

B)

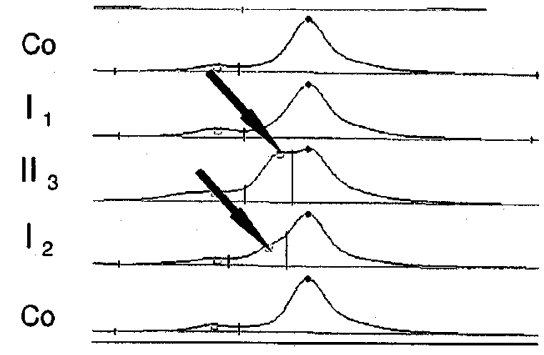

C)

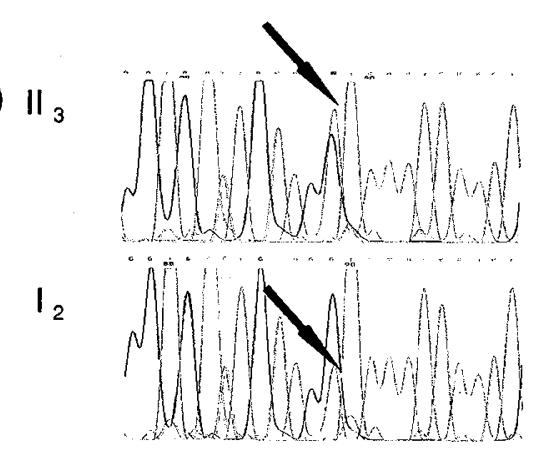

Figure 3 Case $2(1214+1 G \rightarrow A)$. (A) AGS family and symptoms of the affected individuals. Hatched symbol: mosaicism; NR: not reported. (B) SSCP analysis of the exon 5 PCR product with three members of the family; Co: control. Mutation specific shift is indicated by arrows. (C) Sequence data from the antisense strand showing heterozygosity in the patient's and reduced signal intensity in the mother's DNA, respectively (arrows). 


\section{A) 1}
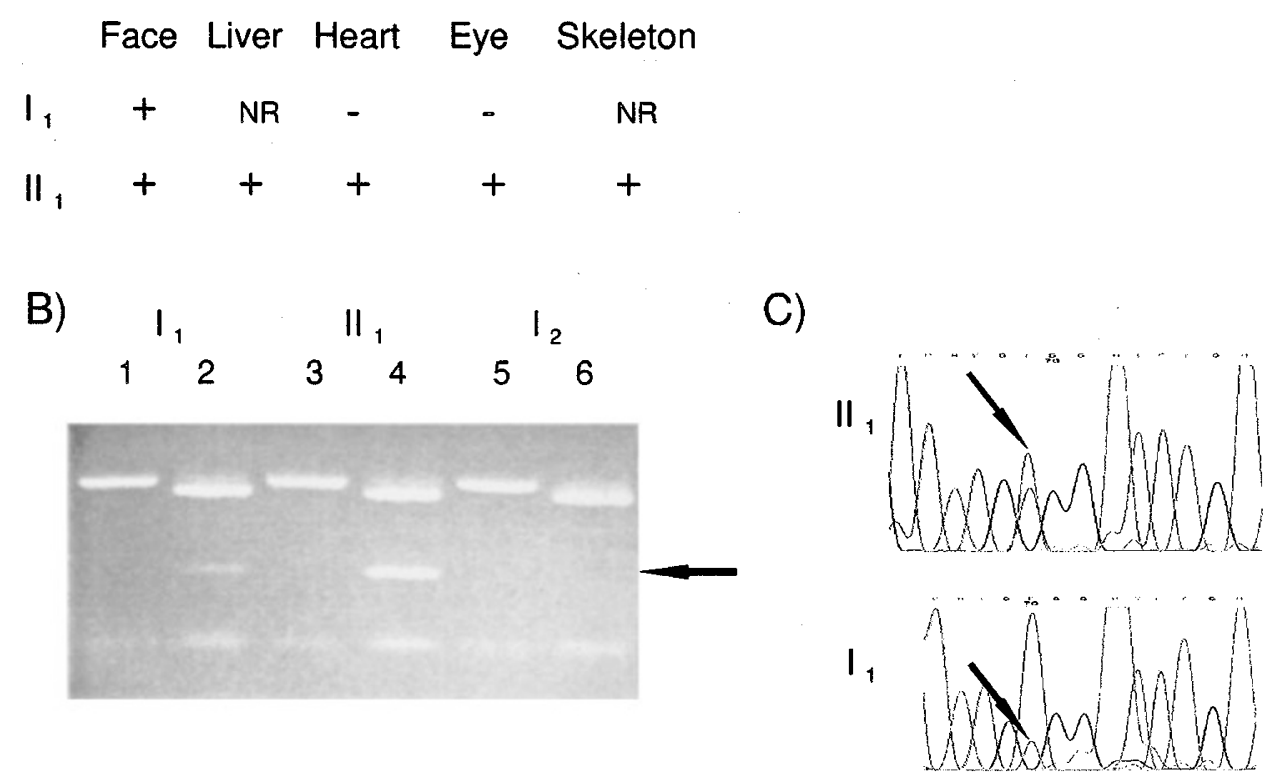

Figure 4 Case $3(\mathrm{R} 184 \mathrm{H})$. (A) AGS family and symptoms of the affected individuals. Hatched symbol: mosaicism; NR: not reported. (B) Lanes 1, 3, 5 PCR product of exon 4 undigested and 2, 4, 6 after digestion using mutation specific endonuclease Pmll. Lanes 1,2: father; 3,4: affected son: 5, 6: unaffected mother. Arrow: mutation specific restriction fragment present in the affected child's and faintly in the father's DNA. (C) Sequence data showing heterozygosity and the mutated nucleotide ' $T$ ' (arrows) in the patient's and weakly in the father's DNA.

appeared with the same facial features as her son without any other sign of AGS. SSCP analysis of the patient did not disclose any abnormal band shift. However, upon deletion screening using microsatellite D20S1154 we observed a signal reduction of the paternal b-allele in the son (Figure $5)$. The observation of the same facial appearance between the mother and the patient seems to reflect a coincidental event. Because of the repeated testing of several blood/ DNA samples, a mixing of the parent's DNA can be excluded. We consider this case to reflect a somatic mosaicism due to a deletion of an undefined portion of JAG1 in the offspring. From comparison of the signal intensity of different alleles at this locus we concluded that the respective deletion affects about $25 \%$ of lymphocytes of the respective patient. To confirm deletion in a mosaic situation FISH analysis was performed on metaphases of the patient using cosmid 3e9 that harbours the entire JAG1 genomic sequence (not shown). Upon analysis of 20 cells we observed in 12 metaphases (60\%) a signal on both chromosomes 20, whereas in eight metaphases (40\%) only one chromosome 20 showed specific FISH signals, which is in agreement with the result of microsatellite analysis (not shown).

\section{Case 5}

A three generation pedigree with six clinically affected individuals, five of whom were investigated by us. SSCP analysis showed a band shift in the exon 10 PCR fragment in the DNA of four affected individuals. Sequencing disclosed a $\mathrm{G} \rightarrow \mathrm{A}$ substitution at cDNA position 1785 resulting in a stop codon (Figure 6). However, we failed to detect this mutation in the grandparents of the pedigree. Because in the second generation, both daughter and son are clinically affected, a de novo event is unlikely. Although the family situation is rather clear, the paternity of the family was confirmed by genotyping using five highly informative polymorphic markers. Because paternity could be demonstrated (not shown), a mosaicism in one of the grandparents is likely. In particular, the grandmother is supposed to have at least gonadal mosaicism, because she demonstrated the facial appearance of other members of the family with AGS. She did not, however, have 
A)
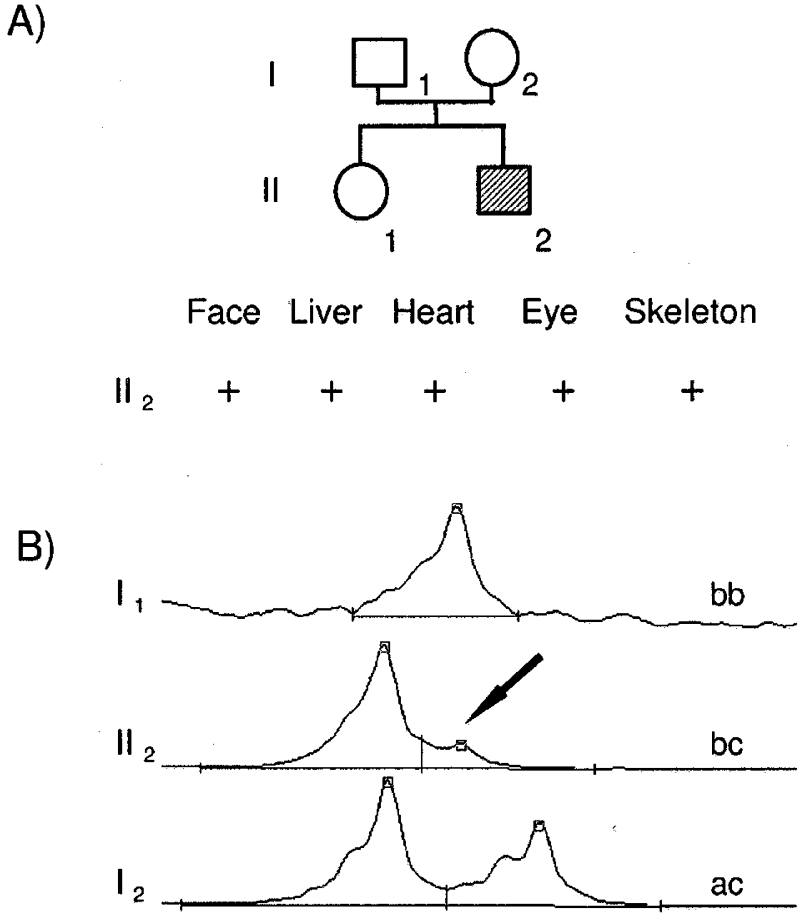

Figure 5 Case 4. (A) Family and symptoms of the affected patient. Hatched symbol: mosaicism. (B) Deletion analysis using microsatellite D20S1154 from intron 19 of JAG1. Reduction of the paternal $b$-allele in the patient indicating mosaicism of a paternal deletion is shown (arrow).

pulmonary stenosis or liver disease, nor were eye abnormalities or butterfly vertebrae noted.

\section{Discussion}

For proper diagnosis and genetic counselling of families with monogenetic diseases it is of great importance to know about the occurrence of mosaicism. The need to detect mosaicism will be of greater relevance the higher its prevalence is in certain diseases. Due to methodological reasons-a low amount of affected blood cells and the limitation to analyse a variety of tissues - the determination of mosaicism will often reflect a fortuitous event. It has been predicted that a disease with a high rate of de novo mutations will be characterised by a high number of mosaics, ${ }^{18}$ which has been confirmed in the case of neurofibromatosis type $2 .{ }^{19}$ Considering that AGS mutations are predominantly sporadic, ${ }^{1}$ (and own unpublished results) mosaicism should occur to a considerable extent, and mosaicism for cytogenetically visible deletions in a few AGS cases has been reported already. $^{20,21}$ As yet, the frequency of mosaicism for minor DNA mutations and/or point mutations has not been investigated.

Our data firstly provide evidence that the frequency of mosaicism for minor DNA mutations and/or point mutations in AGS will account for at least $8.2 \%$ of the cases $(5 / 61)$. However, since the DNA samples included in our analysis were isolated exclusively from lymphocytes, our determination of mosaicism frequency is rather conservative.

Further, we specifically searched for mosaicism in the families of our patients because several siblings of healthy parents were clinically affected. Among 51 families, four $(7.8 \%)$ cases were detected where one of the parents presented a mosaic situation of the mutation which was previously found in the patient.

The degree of mosaicism in different diseases can vary significantly, as reviewed by Zlotogora. ${ }^{22}$ In Apert syndrome (FGRF2) and in Achondroplasia (FGRF3) no mosaic was observable, while $11-20 \%$ of the cases with hemophilia A/B, Duchenne muscular dystrophy or facioscapulohumeral dystrophy were found to represent mosaics. Accordingly, AGS belongs to a group of diseases where mosaicism is found rather frequently. These differences are of importance, particularly in view of genetic counselling and determination of the recurrence risk, since after a diseased child is born to clinically unaffected parents the frequency of mosaicism will reflect the chance of the parents of having another affected child.

Furthermore, the differences in mosaic frequency between different groups of diseases may reflect the degree of clinical variability of the different groups. In the case of neurofibromatosis type I, somatic mosaicism is rather rare, perhaps because such a mosaicism causes clinical symptoms. ${ }^{22}$ Interestingly, the clinically mildest phenotype in our study, ie a typical face (case 3) and the facial appearance in association with an embryotoxon posterior (cases 1 and 2), respectively, presents in three out five individuals where mosaicism is detected (Figures 2-4). Case 5 also presents the typical face but eye abnormalities are not reported (for case 4 see later). The question is whether mosaicism will add to the known variable expressivity of AGS, depending on the proportion of mutation carrying cells. Also, the severity of a phenotype may depend on the type of tissue that is affected. Unfortunately, because no other patient's tissue for investigation was at our disposal, we do not have any data about the involvement of other cell types except for lymphocytes. However, because mutation carrying cells of the patients were detected to a low extent (eg case 1) or not at all (case 5), respectively, blood cells may not exert a relevant AGS causing function. This is also supported by the fact that, despite mosaicism, case 4 presents a complete AGS syndrome. In contrast, mutations affecting fibroblasts may have a greater impact on the severity of the AGS phenotype, yet this observation may also be due to a relatively high ratio of mutated $v s$ normal cells of 1:2. ${ }^{21}$ Mutation studies, including as many tissues as possible of mosaic AGS patients, are necessary to elucidate the role of tissue mosaicism in phenotype development.

Finally, it may be considered whether the high failure rate in mutation detection in AGS patients of about $35 \%{ }^{1,14}$ (own 
A)

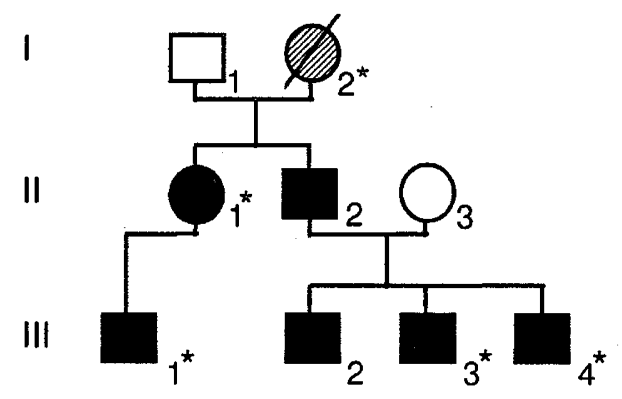

\begin{tabular}{lllll}
\multicolumn{2}{r}{ Face Liver Heart } & Eye & Skeleton \\
$\mathrm{I}_{1}-$ & - & - & - & - \\
$\mathrm{I}_{2}+$ & - & - & $\mathrm{NR}$ & $\mathrm{NR}$ \\
$\mathrm{II}_{1}$ no data & & & \\
$\mathrm{III}+$ & + & + & $\mathrm{NR}$ & $\mathrm{NR}$ \\
$\mathrm{II}_{3}+$ & + & + & + & $\mathrm{NR}$ \\
$\mathrm{III}_{4}+$ & + & + & + & $\mathrm{NR}$
\end{tabular}

B)

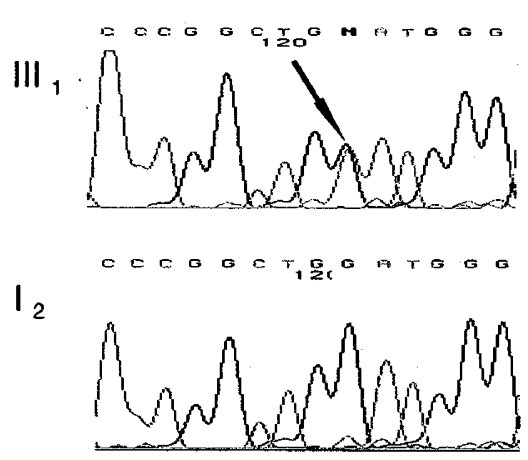

Figure 6 Case 5 (W442X). (A) Three generation pedigree with AGS and symptoms of the affected individuals. Hatched symbol: presumed mosaicism due to facial appearance; NR: not reported. Asterisks indicate individuals who were included in the mutation analysis. (B) Sequence data showing mutation $\mathrm{G} \rightarrow \mathrm{A}$ at $\mathrm{CDNA}$ position 1785 of individual $\mathrm{III}_{1}$ (arrow) and normal sequence of the

unpublished results) may at least in part be due to mosaicism. In addition, because mosaicism may be associated with a very mild phenotype ${ }^{23}$ the appropriate diagnosis can be complicated. Since our data suggest that the prevalence of parental mosaicism in AGS will account for at least $7.8 \%$ of the cases, this type of mutation represents a relevant entity which has to be taken into account in diagnosis, genetic counselling and prognosis in AGS.

\section{Acknowledgements}

We gratefully acknowledge the technical assistance of Mechthild Gräber and the support of many physicians who provided AGS samples and clinical data. We especially want to express our gratitude to $\mathrm{Dr} G$ Matthijs for the mediation of AGS case 4. This study was supported by the DFG.

\section{References}

1 Crosnier C, Driancourt C, Raynaud N et al: Mutations in Jagged1 gene are predominantly sporadic in Alagille Syndrome. Gastroenterology 1999; 116: 1141-1148.

2 Dhorne-Pollet S, Deleuze J-F, Hadchouel M, Bonaiti-Pellié C: Segregation analysis of Alagille syndrome. J Med Genet 1994; 31: $453-457$.
3 Alagille D, Estrada A, Hadchouel M, Gautier M, Odièvre M, Dommergues JP: Syndromic paucity of interlobular bile ducts (Alagille syndrome or arteriohepatic dysplasia): review of 80 cases. J Pediatr 1987; 110: $195-200$.

4 Elmslie FV, Vivian AJ, Gardiner H, Hall C, Mowat AP, Winter RM: Alagille syndrome: Family studies. J Med Genet 1995; 32: $264-$ 268.

5 Byrne JLB, Harrod MJE, Friedman JM, Howard-Peebles PN: del(20p) with manifestations of arteriohepatic dysplasia. Am J Med Genet 1986; 24: 673-678.

6 Schnittger S, Höfers C, Heidemann P, Beermann F, Hansmann I: Molecular and cytogenetic analysis of an interstitial 20p deletion associated with syndromic intrahepatic ductular hypoplasia (Alagille syndrome). Hum Genet 1989; 83: 239-244.

7 Anad F, Burn J, Matthews D et al: Alagille syndrome and deletion of 20p. J Med Genet 1990; 27: $729-737$.

8 Zhang F, Deleuze J-F, Aurias A et al: Interstitial deletion of the short arm of chromosome 20 in arteriohepatic dysplasia (Alagille syndrome). J Pediatr 1990; 116: $73-77$.

9 Hol FA, Hamel BCJ, Geurds MPA et al: Localization of Alagille syndrome to 20p11.2-p12 by linkage analysis of a three generation family. Hum Genet 1995; 95: 687-690.

10 Franke Y: Physikalische Kartierung der Alagille Syndrom Chromosomenregion 20p11.23-12.1 und Suche nach transkriptionell aktiven Sequenzen. PhD thesis, Göttingen, 1996, Univ Diss, ISBN 3-89588-807-9.

$11 \mathrm{Li} \mathrm{L}$, Krantz ID, Deng Y et al: Alagille syndrome is caused by mutations in human Jagged1, which encodes a ligand for Notch1. Nat Genet 1997; 16: 243-251. 
12 Oda T, Elkahloun AG, Pike BL et al: Mutations in the human Jagged1 gene are responsible for Alagille syndrome. Nat Genet 1997; 16: $235-242$.

13 Artavanis-Tsakonas S, Matsuno K, Fortini ME: Notch signaling. Science 1995; 268: $225-232$.

14 Krantz ID, Colliton RP, Genin A et al: Spectrum and frequency of Jagged1 (JAG1) mutations in Alagille syndrome patients and their families. Am J Hum Genet 1998; 62: 1361 - 1369.

15 Yuan Z-R, Kohsaka T, Ikegaya T et al: Mutational analysis of the Jagged1 gene in Alagille syndrome families. Hum Molec Genet 1998; 7: $1363-1369$.

16 Giannakudis I, Stankiewicz P, Röpke A et al: Alagille syndrome due to mutations within the JAG1 gene. Eur J Hum Genet 1998; 6(Suppl 1): 153.

17 Pinkel D, Landegent J, Collins C et al: Fluorescence in situ hybridization with human chromosome-specific libraries: Detection of trisomy 21 and translocation of chromosome 4 . Proc Natl Acad Sci 1988; 85: 9138-9142.
18 Hall JG: Somatic mosaicism: observations related to clinical genetics. Am J Hum Genet 1988; 43: 355-363.

19 Kluwe L, Mautner V-F: Mosaicism in sporadic neurofibromatosis 2 patients. Hum Molec Genet 1998; 7: 2051-2055.

20 Silengo MC, Lopez-Bell G, Biagioli M, Franceschini P: Partial deletion of the short arm of chromosome 20: 46,XXdel (20) (p11)/46,XX mosaicism. Clinic Genet 1988; 33: 108-110.

21 Fryns JP, Kleczkowska A, Decock P, Massa G, van den Berghe $\mathrm{H}$ : $46, \mathrm{XX} / 46 \mathrm{XX}$, del $(20)($ pter $\rightarrow$ p12.2) mosaicism limited to fibroblasts associated with MCA/MR and severe growth defect. Ann Genet 1992; 35: 234-236.

22 Zlotogora J: Germ line mosaicism. Hum Genet 1998; 102: 381 386.

23 Plant K, Boye E, Green PM, Vetrie D, Flinter FA: Somatic mosaicism associated with a mild Alport syndrome phenotype. J Med Genet 2000; 37: 238-239. 